\title{
On the diagonal weights of inscribed polytopes
}

\author{
Jin Akiyama and Ikuro Sato \\ Jin Akiyama received a D.Sc. from Tokyo University of Science for his work in graph \\ theory and combinatorics. He is now the director of the Research Center for Science \\ and Math Education at Tokyo University of Science and also serves as the founding \\ editor of the journal of Graphs and Combinatorics. He is interested in graph theory, \\ discrete and computational geometry, and also in mathematics education. \\ Ikuro Sato graduated from Tohoku University School of Medicine in 1981, and re- \\ ceived a M.D. in pathology. He is working at the Research Institute of Miyagi Cancer \\ Center as the director and clinical professor of pathology. He has also published many \\ mathematical papers on polytopes in higher dimensions.
}

\section{The diagonal weight}

For a given polytope $\Pi$ with $v$ vertices $P_{1}, P_{2}, \ldots, P_{v}$, we define the diagonal weight of $\Pi$ as the sum of the squares of the lengths of all diagonals and sides of $\Pi$, and denote it by $\alpha(\Pi)$. That is, the diagonal weight $\alpha(\Pi)$ of $\Pi$ is defined as follows:

$$
\alpha(\Pi)=\sum_{i, j}\left|P_{i} P_{j}\right|^{2}
$$

where $\left|P_{i} P_{j}\right|$ is the distance between $P_{i}$ and $P_{j}$, and the sum is taken over all possible pairs of $P_{i}$ and $P_{j}$

We first consider a few regular polygons on the plane, namely, the equilateral triangle, the square, the regular pentagon and the regular hexagon inscribed in unit circles as illustrated in Figure 1.

Berechnet man von einem dem Einheitskreis einbeschriebenen regulären $n$-Eck die Summe der Quadrate der Seitenlängen und Diagonalen, so erhält man $n^{2}$. Hätten Sie's gewusst? Die Autoren der vorliegenden Arbeit haben genauer hingeschaut und bemerkt, dass diese Eigenschaft in beliebigen Dimensionen $m \geq 2$ für alle der Einheitssphäre einbeschriebenen Polytope mit $n$ Ecken gilt, wenn der Eckenschwerpunkt mit dem Zentrum der Umkugel zusammenfällt. Die Autoren untersuchen auch die Frage, welche dreidimensionalen Polyeder, deren Seiten reguläre Vielecke sind, diese Schwerpunktsbedingung erfüllen. 
$\mathrm{T}$ :
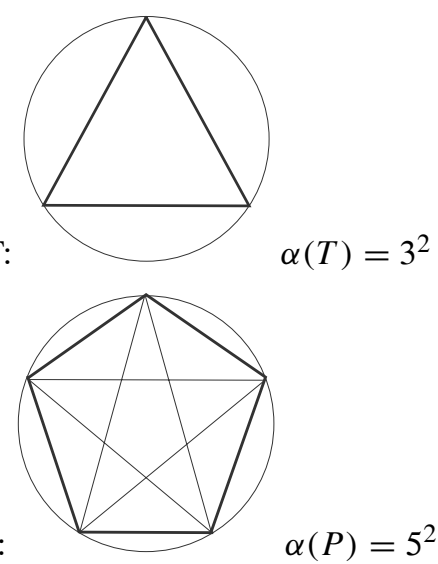

S:

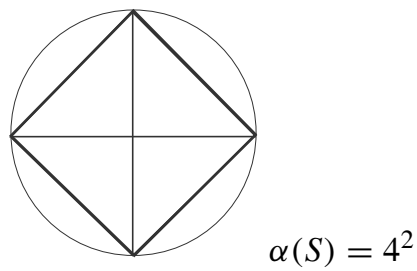

$\mathrm{H}:$

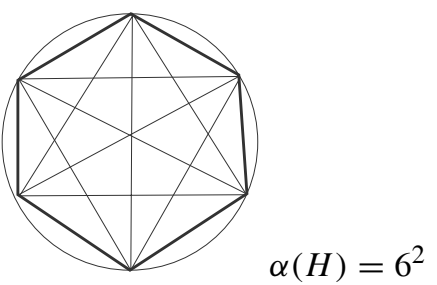

Fig. 1

Then it follows at once that the diagonal weights of these regular polygons are 9, 16, 25 and 36, respectively. This simple observation suggests to us that the diagonal weight $\alpha(\Pi)=v^{2}$ holds for every regular polygon $\Pi$ with $v$ vertices.

Figure 2 illustrates two of the polyhedra in 3-dimensional space: the cube and the regular octahedron. The following theorem shows that the result holds not only on the plane but also in any higher dimension $n(n \geq 2)$.

C:

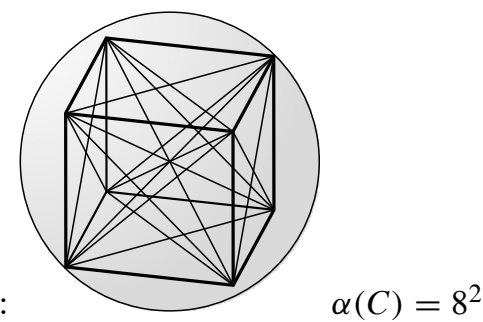

$\mathrm{O}$ :

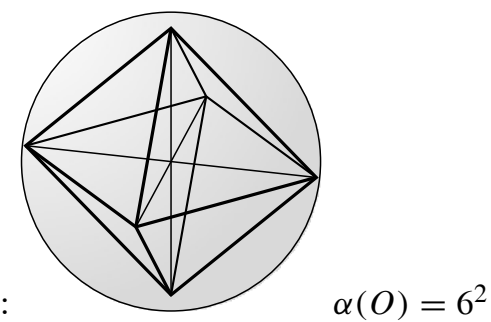

Fig. 2

Theorem 1. Let $R$ be a regular $n$-dimensional polytope with $v$ vertices $P_{1}, P_{2}, \ldots, P_{v}$ which is inscribed in a unit $n$-sphere. Then the diagonal weight $\alpha(R)$ is $v^{2}$ for every dimension $n \geq 2$.

Proof. Let

$$
\begin{aligned}
Q & =\sum_{j=1}^{v}\left|P_{1} P_{j}\right|^{2} \\
& =\left(\boldsymbol{p}_{1}-\boldsymbol{p}_{1}\right) \cdot\left(\boldsymbol{p}_{1}-\boldsymbol{p}_{1}\right)+\left(\boldsymbol{p}_{2}-\boldsymbol{p}_{1}\right) \cdot\left(\boldsymbol{p}_{2}-\boldsymbol{p}_{1}\right)+\cdots+\left(\boldsymbol{p}_{v}-\boldsymbol{p}_{1}\right) \cdot\left(\boldsymbol{p}_{v}-\boldsymbol{p}_{1}\right),
\end{aligned}
$$


where each $\boldsymbol{p}_{j}$ is a position vector of $P_{j}$ in relation to the center of $R$. Since

$\left(\boldsymbol{p}_{j}-\boldsymbol{p}_{1}\right) \cdot\left(\boldsymbol{p}_{j}-\boldsymbol{p}_{1}\right)=\boldsymbol{p}_{1} \cdot \boldsymbol{p}_{1}-2 \boldsymbol{p}_{1} \cdot \boldsymbol{p}_{j}+\boldsymbol{p}_{j} \cdot \boldsymbol{p}_{j}, \quad \boldsymbol{p}_{1} \cdot \boldsymbol{p}_{1}=1 \quad$ and $\quad \boldsymbol{p}_{j} \cdot \boldsymbol{p}_{j}=1$,

we have

$$
\left(\boldsymbol{p}_{j}-\boldsymbol{p}_{1}\right) \cdot\left(\boldsymbol{p}_{j}-\boldsymbol{p}_{1}\right)=2-2 \boldsymbol{p}_{1} \cdot \boldsymbol{p}_{j}
$$

Thus we obtain

$$
Q=2 v-2 \boldsymbol{p}_{1} \cdot\left(\boldsymbol{p}_{1}+\boldsymbol{p}_{2}+\cdots+\boldsymbol{p}_{v}\right) .
$$

Since the centroid of the vertices of $R$ is the origin,

$$
\boldsymbol{p}_{1}+\boldsymbol{p}_{2}+\cdots+\boldsymbol{p}_{v}=\mathbf{0}
$$

and hence

$$
Q=2 v
$$

Since the length of each diagonal occurs twice in $v \cdot Q$,

$$
\alpha(R)=\frac{v}{2} \cdot Q=v^{2}
$$

The argument above holds for every $n$-dimensional polytope whose centroid is the origin.

There are many applications of this theorem. For instance, the diagonal weight of a regular 4-dimensional 120 -cell is $600^{2}$, since it has 600 vertices. Likewise, it is straight forward to find the length $d_{n}$ of a side of an $n$-simplex inscribed in a unit $n$-sphere, since $\left(\begin{array}{c}n+1 \\ 2\end{array}\right) d_{n}^{2}=$ $(n+1)^{2}$.

Corollary 1. Let $P$ be an n-polytope inscribed in a unit $n$-sphere and having the property that $\sum \boldsymbol{p}_{j}=\mathbf{0}$. Then the diagonal weight $\alpha(P)=v^{2}$ holds for $P$.

\section{Well-balanced polyhedra}

We next generalize Theorem 1 slightly for a polytope whose centroid does not coincide with its circumcenter.

Theorem 2. Let $\Pi$ be an n-polytope with $v$ vertices $P_{1}, P_{2}, \ldots, P_{v}$ inscribed in a unit $n$-sphere and $c$ be the distance between the centroid and the circumcenter of $\Pi$. Then we have

$$
\alpha(\Pi)=v^{2}\left(1-c^{2}\right), \quad \text { where } 0 \leqq c \leqq 1 .
$$

Proof. Let $\boldsymbol{c}$ be the vector defined as $\boldsymbol{c}=\left(\boldsymbol{p}_{1}+\boldsymbol{p}_{2}+\cdots+\boldsymbol{p}_{v}\right) / v$, and $c$ be its norm, i.e, $c=|c|$.

As $Q_{j}=2 v\left(1-\boldsymbol{p}_{j} \cdot \boldsymbol{c}\right)$, we have $\alpha(\Pi)=1 / 2 \sum Q_{j}=v^{2}\left(1-c^{2}\right), 0 \leqq c \leqq 1$.

Theorem 2 implies that $\alpha(\Pi) \leqq v^{2}$ for every inscribed $n$-polytope with $v$ vertices. An inscribed $n$-polytope $P$ with $v$ vertices is said to be well-balanced if $P$ satisfies $\alpha(P)=v^{2}$. That is, the diagonal weight of a balanced polytope attains the upper bound. 
In the 3-dimensional case, there are infinitely many convex well-balanced polyhedra other than the Platonic solids. Our next purpose is to determine all well-balanced convex polyhedra with regular polygons as faces.

All convex polyhedra with regular polygons as their faces are known. There are five Platonic solids, thirteen Archimedean solids, the two infinite sequences of prisms and antiprisms, and 92 other polyhedra discovered by N.W. Johnson. We will denote Johnson's polyhedra by $\mathrm{J} 1$ to J92, following Johnson's numbering in his original paper [2]. Drawings and nets of other solids may be viewed on Wikipedia. We checked all such polyhedra with the property that their circumcenters coincide with the centroids on the basis of their coordinates.

Corollary 2. The following are all the well-balanced convex polyhedra with regular polygons as faces:

all Platonic solids, all Archimedean solids, all Archimedean prisms,

all Archimedean antiprisms, J27, J34, J37, J72, J73, J74, J75 and J80.

\title{
Acknowledgement
}

The authors thank Dr. Nobuaki Muto for computing the centroid of every Johnson polyhedron by computer search.

\section{References}

[1] Maehara, H.: Geometry on Circles and Spheres. Asakura Shoten (1998), in Japanese.

[2] Johnson, N.W.: Convex solids with regular faces. Canad. J. Math. 18 (1966), 169-200.

\author{
Jin Akiyama \\ Research Center for Science \\ and Math Education \\ Tokyo University of Science \\ e-mail: ja@jin-akiyama.com \\ Ikuro Sato \\ Department of Pathology \\ Research Institute \\ Miyagi Cancer Center, Japan \\ e-mail: sato-ik510@miyagi-pho.jp
}

